\title{
Andrographolide induces Nrf2 and heme oxygenase 1 in astrocytes by activating p38 MAPK and ERK
}

\author{
Siew Ying Wong ${ }^{1}$, Michelle G. K. Tan ${ }^{1,2}$, Peter T. H. Wong ${ }^{1}$, Deron R. Herr ${ }^{1}$ and Mitchell K. P. Lai ${ }^{\text {** }}$
}

\begin{abstract}
Background: Andrographolide is the major labdane diterpenoid originally isolated from Andrographis paniculata and has been shown to have anti-inflammatory and antioxidative effects. However, there is a dearth of studies on the potential therapeutic utility of andrographolide in neuroinflammatory conditions. Here, we aimed to investigate the mechanisms underlying andrographolide's effect on the expression of anti-inflammatory and antioxidant heme oxygenase-1 (HO-1) in primary astrocytes.

Methods: Measurements of the effects of andrograholide on antioxidant HO-1 and its transcription factor, Nrf2, include gene expression, protein turnover, and activation of putative signaling regulators.

Results: Andrographolide potently activated Nrf2 and also upregulated HO-1 expression in primary astrocytes. Andrographolide's effects on Nrf2 seemed to be biphasic, with acute (within 1 h) reductions in Nrf2 ubiquitination efficiency and turnover rate, followed by upregulation of Nrf2 mRNA between 8 and $24 \mathrm{~h}$. The acute regulation of Nrf2 by andrographolide seemed to be independent of Keap1 and partly mediated by p38 MAPK and ERK signaling.

Conclusions: These data provide further insights into the mechanisms underlying andrographolide's effects on astrocyte-mediated antioxidant, and anti-inflammatory responses and support the further assessment of andrographolide as a potential therapeutic for neurological conditions in which oxidative stress and neuroinflammation are implicated.
\end{abstract}

Keywords: Andrographolide, Nrf2, Heme oxygenase 1, Astrocyte, Antioxidant response, Mitogen-activated protein kinases

\section{Background}

Oxidative stress goes hand in hand with inflammation and their underlying mechanisms are inextricably interconnected [1]. Growing evidence suggest that oxidative stress and neuroinflammation underpin a diverse range of CNS diseases, including stroke, traumatic brain injury, multiple sclerosis, Alzheimer's, Parkinson's, and other neurodegenerative diseases [2-5]. The brain is particularly vulnerable to oxidative stress due to an abundance of iron and polyunsaturated fatty acids which are susceptible to lipid peroxidation $[6,7]$, thus underscoring the

\footnotetext{
* Correspondence: mitchell.lai@dementia-research.org

${ }^{1}$ Department of Pharmacology, Yong Loo Lin School of Medicine, National University of Singapore, Unit 09-01, Centre for Translational Medicine (MD6) 14 Medical Drive, Kent Ridge 117599, Singapore

Full list of author information is available at the end of the article
}

importance of maintaining redox balance for normal brain functioning. One mechanism by which the brain defends itself against oxidative insults is via the upregulation of antioxidant molecules. Interestingly, oxidative stress strongly induces expression of heme oxygenase-1 (HO-1) in the CNS, a system not actively involved in red blood cell metabolism [8]. Indeed, while HO-1's primary function is the catalysis of heme, leading to the eventual formation of antioxidant bilirubin, carbon monoxide, and ferrous iron $\left(\mathrm{Fe}^{2+}\right)$, and in the process, prevents heme-mediated free radical production [9], HO-1 is also found to have anti-neuroinflammatory and neuroprotective properties in the CNS $[8,10]$. Furthermore, HO-1 upregulation has been reported to be a mechanism by which certain isoflavone metabolites protect astrocytes 
from hydrogen peroxide-induced reactive oxygen species [11]. Together with their well-recognized roles in facilitating neuronal trophic support, biochemical homeostasis, blood brain barrier integrity, response to neuroinflammatory signals and scar formation, astrocytes play a critical role in redox homeostasis and are the major source of antioxidant molecules and enzymes which protect them and the neurons they support from oxidative stresses $[12,13]$. The critical involvement of astrocytes in neuroinflammation and oxidative stress protection as well as the pathogenicity of astrocyte dysregulation in various CNS diseases [14-16] gave research impetus to discover and characterize novel antineuroinflammatory/antioxidant therapeutics with efficacy on astrocytes. Besides the aforementioned work on isoflavone metabolites [11], a wide range of other bioactive molecules have been studied. Of these, andrographolide is a labdane diterpenoid derived from the herbaceous Andrographis paniculata, which has been traditionally used in Asia to treat a variety of ailments, including fever, cough, tuberculosis, snake bites, respiratory tract, and urinary tract infections [17, 18]. Andrographolide has been reported to exhibit antiinflammatory and antioxidant activities in peripheral tissues [19]. Furthermore, we have previously shown that the brain-penetrant andrographolide attenuates IL- $1 \beta$ or lipopolysaccharide-stimulated upregulation of the $\mathrm{C}-\mathrm{C}$ and $\mathrm{C}-\mathrm{X}-\mathrm{C}$ chemokines in the brain cortex as well as in cultured astrocytes $[20,21]$. However, while the efficacy of andrographolide in reducing oxidative stress in the CNS has been demonstrated in several studies [22-24], the underlying molecular mechanisms have not been thoroughly ascertained. Furthermore, andrographolide seems to have pleiotropic effects on signaling pathways involved in inflammatory and oxidative stress responses, but the mechanisms underlying these effects appear different in various cell types [20,21, 25-27]. In this study, our focus is to investigate the effects of andrographolide on $\mathrm{HO}-1$ expression in astrocytes. Because $\mathrm{HO}-1$ is a known gene target of transcription factor NF-E2-related factor 2 (Nrf2), which is critically involved in cellular defense against oxidative stress [11, 28, 29], we also studied andrographolide effects on astroglial Nrf2 regulation.

\section{Methods}

\section{Reagents}

Andrographolide ( $\geq 98 \%$ purity, see Fig. 1a), cycloheximide (CHX), and other chemical reagents were purchased from Sigma-Aldrich Ltd. (St. Louis, MO, USA) unless otherwise specified. Various concentrations of andrographolide, along with mitogen-activated protein kinase (MAPK) inhibitors SB202190 and PD98059 (Tocris Bioscience, Bristol, UK) were dissolved in up to $0.1 \%$ dimethyl sulfoxide (DMSO) for primary astrocyte treatment. 0.1\% DMSO was also used as a control (vehicle) in the cell-based assays.

\section{Primary astrocyte culture}

Primary astrocyte cultures for in vitro assays were obtained from newborn Sprague Dawley rat pups (postnatal days 1-3) as previously described [20]. Briefly, isolated cortices were separated from the meninges in PBS, dissociated with $0.25 \%$ trypsin-EDTA (ThermoFisher Scientific, Waltham, MA, USA) and filtered through a $40-\mu \mathrm{m}$ nylon cell strainer (BD Falcon, Franklin Lakes, NJ, USA). The resultant filtrate was centrifuged and resuspended in DMEM/F12 media supplemented with $10 \%$ heat-inactivated fetal bovine serum (FBS), $100 \mathrm{U} / \mathrm{mL}$ penicillin, and $100 \mathrm{mg} / \mathrm{mL}$ streptomycin (all from ThermoFisher Scientific, Waltham, MA, USA). After 79 days of culture in a humidified atmosphere with $5 \%$ $\mathrm{CO}_{2}$ at $37^{\circ} \mathrm{C}$, astrocytes were enriched by vigorously shaking the cell culture flasks at $350 \mathrm{rpm}$ to remove nonadherent microglial cells and oligodendrocytes. Immunofluorescence staining with antibodies to glial fibrillary acidic protein indicated the purity of the resultant astrocyte cultures to be $>95 \%$ (data not shown).

\section{Cell viability assays}

Rat primary astrocytes were plated onto 24-well tissue culture plates at a density of $1 \times 10^{5}$ cells per well and treated with various concentrations of andrographolide $(0-100 \mu \mathrm{M})$ for $24 \mathrm{~h}$. Cell concentrations and viability (recorded as \% viable cells) were determined with the Muse $^{\mathrm{rm}}$ Cell Analyzer (Merck Millipore, Darmstadt, Germany) according to manufacturer's instructions.

\section{Subcellular fractionation}

Subcellular fractionation was performed using Nuclear Extract kit according to manufacturer's instructions (Active Motif, Tokyo, Japan). Briefly, media was aspirated out from $10-\mathrm{cm}$ culture dishes and the cells washed with $5 \mathrm{ml}$ ice-cold phosphate-buffered saline (PBS) with PhosSTOP ${ }^{\mathrm{mx}}$ phosphatase inhibitor cocktail from Roche Life Science (Penzberg, Germany) and then transferred to a prechilled conical tube. Subsequently, the cell suspension was centrifuged at $200 \times g$ for $5 \mathrm{~min}$ at $4{ }^{\circ} \mathrm{C}$, and the resulting pellet re-suspended in hypotonic buffer on ice, followed by addition of detergent and vortexing to obtain cell lysis (checked under microscope). After further centrifugation $\left(14,000 g, 30 \mathrm{~s}, 4{ }^{\circ} \mathrm{C}\right)$, the supernatant (cytoplasmic fraction) was transferred into new tubes while the pellet was re-suspended in proprietary lysis buffer. Samples were incubated for $30 \mathrm{~min}$ before centrifugation $\left(14,000 \mathrm{~g}, 10 \mathrm{~min}, 4^{\circ} \mathrm{C}\right)$. The resulting supernatant (nuclear fraction) was transferred to new tubes and stored frozen until use. 

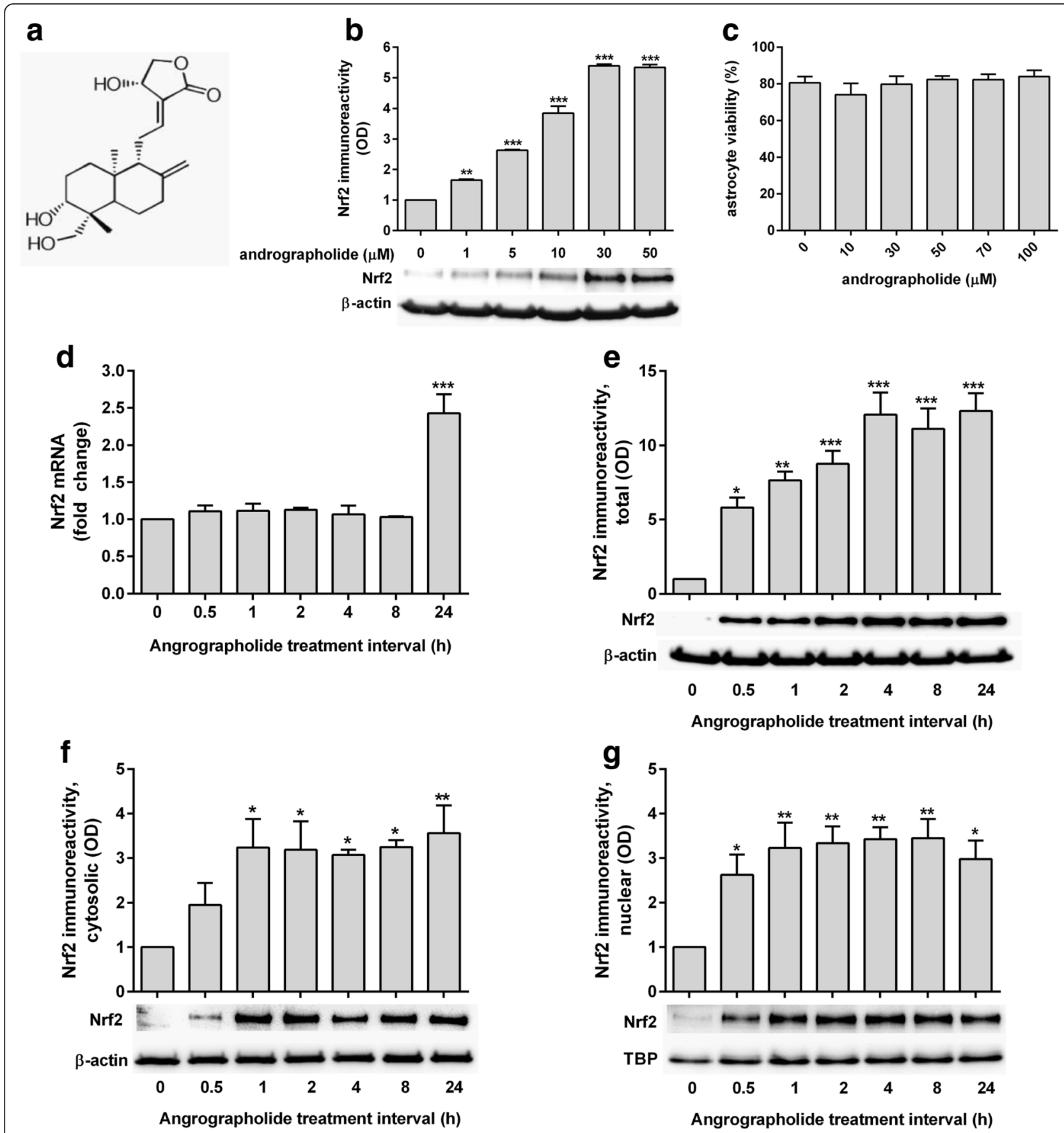

Fig. 1 Dose and time-course of andrographolide's effects on Nrf2. a Structure of andrographolide: 3-[2-[decahydro-6-hydroxy-5-(hydroxymethyl)5,8a-dimethyl-2-methylene-1-napthalenyl]ethylidene]dihydro-4-hydroxy-2(3H)-furanone (CAS no. 5508-58-7). b Bar graphs depict mean \pm S.E.M. Nrf2 immunoreactivities (optical density, OD fold changes normalized to $\beta$-actin) in rat primary astrocytes treated with andrographolide at the indicated concentrations for $1 \mathrm{~h}$ (with representative immunoblot of 3 independent experiments), with the vehicle-only ("0 $\mu \mathrm{M}$ ") group set at 1. c Cell viability (in mean \pm S.E.M. \% of vehicle-only from three independent experiments) of primary astrocytes after $24 \mathrm{~h}$ treatment with andrographolide at the indicated concentrations. $\mathbf{d}$ mRNA and $\mathbf{e}$ immunoreactivity changes of Nrf2 in primary astrocytes treated with andrographolide (50 $\mu \mathrm{M})$ for the indicated time intervals, together with immunoreactivites in the $\mathbf{f}$ cytosolic and $\mathbf{g}$ nuclear fractions (with representative immunoblots). Bar graphs show mRNA or immunoreactivity expressed as mean \pm S.E.M. fold change in transcript level or optical density (OD), respectively, with vehicle-only ("0 $h$ ") group set as 1, from three to four independent experiments. Raw transcript values were normalized to mean expression of housekeeping genes (see the "Methods" section) prior to conversion to fold-change values while immunoreactivities were normalized to $\beta$-actin except for Nrf2 nuclear fractions, which were normalized to TATA-binding protein (TBP). ${ }^{*} p<0.05 ;{ }^{* *} p<0.01$; and ${ }^{* *} p<0.001$; significantly different from vehicle-only group (one-way ANOVA with Dunnett's post hoc tests) 


\section{Real-time PCR}

For measurements of Nrf2 and HO-1 messenger RNA (mRNA) expression, treated astrocytes were lysed in TRIzol $^{\circ}$ reagent (ThermoFisher Scientific, Waltham, MA, USA), precipitated, and then processed for RNA isolation according to manufacturer's instructions (NucleoSpin ${ }^{\bullet}$ RNA kit from Macherey-Nagel GmbH, Düren, Germany). After the assessment of RNA concentration and purity with a NanoDrop spectrophotometer (ThermoFisher Scientific, Waltham, MA, USA), complementary DNA (cDNA) was synthesized from RNA samples using high-capacity cDNA reverse transcriptase kits and quantitative real-time PCR was performed using a Applied Biosystems ${ }^{\circ}$ StepOnePlus ${ }^{\text {tu }}$ Real-Time PCR instrument (ThermoFisher Scientific, Waltham, MA, USA). Table 1 lists the primer sequences of the genes of interest, and fold-change values of gene expression relative to vehicle-treated controls were computed for each experimental group using the $2^{-\Delta \Delta C T}$ formula, after the normalization against the geometric mean of GAPDH and $\beta$-actin expression.

\section{Immunofluorescence imaging}

Astrocytes plated on glass coverslips were treated with andrographolide, then fixed with $4 \%$ paraformaldehyde/
PBS for 15 min, and washed thrice with PBS followed by incubation with permeabilizing buffer containing $0.1 \%$ Triton-X100 in PBS for 5 min at $25^{\circ} \mathrm{C}$. The cells were then incubated in blocking solution (5 \% BSA in permeabilizing buffer) for $1 \mathrm{~h}$ before incubation overnight with primary antibodies against HO-1 (1:200 dilution) in blocking solution at $4{ }^{\circ} \mathrm{C}$. Subsequently, cells were washed thrice with PBS and incubated with anti-mouse IgG Alexa Flour ${ }^{\circ} 488$ (1:400 dilution, Cell Signaling Technology, Danvers, MA, USA) for $1 \mathrm{~h}$ at $25^{\circ} \mathrm{C}$. Cells were then washed with PBS before mounting the coverslips onto glass slides using mounting medium containing DAPI nuclear stain (Vector Laboratories, Burlingame, CA, USA). Immunofluorescence images were taken with an Axioplot microscope equipped with Carl Zeiss 510 confocal imaging scan-head and software (Carl Zeiss MicroImaging, Jena, Germany), with the same parameters used for all images.

\section{Immunoblotting}

Astrocytes treated with andrographolide with and without chemical inhibitors were lysed in situ on tissue culture plates by adding boiling Laemmli sample buffer (Bio-Rad, Berkeley, CA, USA), heated further at $95{ }^{\circ} \mathrm{C}$ for $5 \mathrm{~min}$, then allowed to cool before electrophoretic separation on $10 \%$ polyacrylamide gels, transferred onto

Table 1 Primary antibodies and primers used in this study

\begin{tabular}{|c|c|c|c|c|}
\hline Target & Antibody & Format & Dilution $^{a}$ & Source Company \\
\hline $\mathrm{OH}-1$ & $\mathrm{OH}-1$ & Rabbit polyclonal & 1:1000; 1:200 (IF) & Abcam \\
\hline Nrf2 & $C-20$ & Rabbit polyclonal & $1: 1000$ & Santa Cruz \\
\hline pS40-Nrf2 & phos-Nrf2 & Rabbit monoclonal & $1: 1000$ & Abcam \\
\hline Nqo1 & $C-19$ & Goat polyclonal & $1: 1000$ & Santa Cruz \\
\hline Keap1 & $E-20$ & Goat polyclonal & $1: 1000$ & Santa Cruz \\
\hline Total Erk1/2 & Erk1/2 & Rabbit polyclonal & $1: 1000$ & Cell Signaling \\
\hline pT202/pY204-Erk & phos-Erk & Rabbit polyclonal & $1: 1000$ & Cell Signaling \\
\hline Total p38 & p38 & Rabbit polyclonal & $1: 1000$ & Cell Signaling \\
\hline pT180/pY182-p38 & D3F9 & Rabbit monoclonal & $1: 1000$ & Cell Signaling \\
\hline$\beta$-actin & $A C-15$ & Mouse monoclonal & $1: 5000$ & Sigma-Aldrich \\
\hline Lamin B1 & Lamin B1 & Rabbit polyclonal & $1: 10,000$ & Abcam \\
\hline TBP & TBP & Mouse monoclonal & $1: 5000$ & Abcam \\
\hline Ubiquitin & P4D1 & Mouse monoclonal & $30 \mu \mathrm{L}$ per $1 \mathrm{mg}$ lysate protein $(\mathrm{IP})^{\mathrm{b}}$ & Santa Cruz \\
\hline Target & \multicolumn{2}{|c|}{ Forward primer } & \multicolumn{2}{|l|}{ Reverse primer } \\
\hline $\mathrm{OH}-1$ & \multicolumn{2}{|c|}{ 5'-GGCTCTCTITTCTTGGGCCT-3' } & \multicolumn{2}{|l|}{ 5'-GCCTCTACCGACCACAGTTC-3' } \\
\hline Nrf2 & \multicolumn{2}{|c|}{ 5'-CAGTCTTCACCACCCCTGAT-3' } & \multicolumn{2}{|l|}{ 5'-CAGTGAGGGGATCGATGAGT-3' } \\
\hline Nqo1 & \multicolumn{2}{|c|}{ 5'-GCGAGCGGGGAAAATACTCT-3' } & \multicolumn{2}{|l|}{ 5'-CCTCCTGCCCTAAACCACAG-3' } \\
\hline$\beta$-actin & \multicolumn{2}{|c|}{ 5'-ACCCGCGAGTACAACCTTCT-3' } & \multicolumn{2}{|l|}{ 5'-TTCTGACCCATACCCACCAT-3' } \\
\hline GAPDH & \multicolumn{2}{|c|}{ 5'-CTCATGACCACAGTCCATGC-3' } & \multicolumn{2}{|l|}{ 5'-TTCTGACCCATACCCACCAT-3' } \\
\hline
\end{tabular}

${ }^{a}$ Stated dilutions are for immunoblotting except for immunofluorescence (IF) staining and immunoprecipitation (IP). Source companies are Abcam (Cambridge, UK); Cell Signaling Technology (Danvers, MA, USA); Santa Cruz Biotechnology (Dallas, TX, USA); and Sigma-Aldrich (St. Louis, MO, USA)

${ }^{\mathrm{b} C o n j u g a t e d ~ w i t h ~ a g a r o s e ~ b e a d s ~}(500 \mu \mathrm{g}$ antibody $/ 0.25 \mathrm{~mL}$ agarose) 
nitrocellulose membranes (ThermoFisher Scientific, Waltham, MA, USA), and blocked with $5 \%$ non-fat milk in 10 mM PBS, pH7.4 with $0.1 \%$ Tween $^{\circ} 20$ (PBST) at room temperature for $1 \mathrm{~h}$. Membranes were then washed and probed with primary antibody diluted in PBST with $5 \%$ bovine serum albumin overnight at $4{ }^{\circ} \mathrm{C}$. The primary antibodies used are listed in Table 1. Following primary antibody incubation, membranes were washed with PBST, then incubated with horse radish peroxidase (HRP)-conjugated secondary antibodies (goat anti-rabbit, goat antimouse, and donkey anti-goat, respectively, from Jackson ImmunoResearch, West Grove, PA, USA), and diluted at 1:5000 for $1 \mathrm{~h}$ at $25^{\circ} \mathrm{C}$. To detect the proportion of phosphorylated protein, membranes were first probed for phospho-proteins then stripped and reblotted for total proteins. Immunoblots were visualized using HRP substrate (Luminata ${ }^{\mathrm{Tm}}$ Forte or Crescendo, Merck Millipore, Darmstadt, Germany) and quantified by image analyzer (UVItec Ltd., Cambridge, UK).

\section{Immunoprecipitation}

Treated astrocytes were harvested with RIPA buffer (Santa Cruz Biotechnology, Dallas, TX, USA) supplemented with Complete ULTRA $^{\mathrm{m}}$ protease inhibitor tablets and PhosSTOP ${ }^{\mathrm{Tw}}$ phosphatase inhibitor (Roche Life Science, Penzberg, Germany). The resultant lysate was sonicated and centrifuged at $14,000 \times g$ for $10 \mathrm{~min}$ at $4{ }^{\circ} \mathrm{C}$, and supernatant was measured for protein (Pierce $^{\mathrm{Tw}}$ Commassie kit, ThermoFisher Scientific, Waltham, MA, USA). Input samples were prepared by adding $70 \mu \mathrm{g}$ protein in 1:1 ratio to boiling Laemmli sample buffer with further heating at $95^{\circ} \mathrm{C}$ for $5 \mathrm{~min}$. For immunoprecipitation, $1 \mathrm{mg}$ lysate was incubated with antiubiquitin monoclonal antibody-conjugated agarose beads (Santa Cruz Biotechnology, Dallas, TX, USA) for $3 \mathrm{~h}$ at $4^{\circ}$ $\mathrm{C}$ with rotation. Agarose beads were then pelleted and washed four times with ice-cold immunoprecipitation buffer $(20 \mathrm{mM}$ Tris- $\mathrm{HCl} \mathrm{pH} 8,140 \mathrm{mM} \mathrm{NaCl}, 2 \mathrm{mM}$ EDTA, $1 \%$ Triton X-100), before adding Laemmli buffer and boiling. Input samples and immunoprecipitates were electrophoretically resolved on 10 and $8 \%$ polyacrylamide gels, respectively, then transferred onto membranes, and immunoblotted for Nrf2 (see above, and Table 1).

\section{Statistical analyses}

Data analyses were performed using SPSS Statistics software (version 21, IBM Inc., Armonk, NY, USA). Dose effects of andrographolide were compared to untreated controls using analysis of variance (ANOVA) with Dunnett's post hoc tests, while other pair-wise comparisons of the experimental groups were performed using ANOVA followed by Bonferroni's post hoc tests, with $p$ values $<0.05$ considered statistically significant. All experiments were performed independently at least three times.

\section{Results \\ Andrographolide positively regulated Nrf2 in astrocytes}

To assess the potential of andrographolide to induce Nrf2, a known regulator of $\mathrm{HO}-1$ transcription [11, 28, 29], primary astrocytes were treated with various concentrations of andrographolide for $1 \mathrm{~h}$ and immunoblotted with Nrf2 antibody. Interestingly, while the predicted molecular weight of Nrf2 is around $66 \mathrm{kDa}$, there is increasing evidence that the biologically relevant bands fall around 95$110 \mathrm{kDa}$ [30], and this unusual migration pattern of Nrf2 may be due to actin binding or the high acidic residue content of Nrf2 [31, 32]. Indeed, we observed two major bands above the 50 and $100 \mathrm{kDa}$ molecular weight markers in our immunoblots (Additional File 1: Figure S1) and have selected the $\sim 110 \mathrm{kDa}$ bands for analyses. Figure $1 \mathrm{~b}$ shows that andrographolide dose-dependently increased Nrf2 levels from as low as $1 \mu \mathrm{M}$, while astrocyte viability was not significantly affected with up to $100 \mu \mathrm{M}$ andrographolide for $24 \mathrm{~h}$ (Fig. 1c).

\section{Independent time-course of andrographolide's effects on Nrf2 mRNA versus protein in astrocytes}

Next, we studied the time-course of both Nrf2 mRNA and protein changes in andrographolide-treated astrocytes. Interestingly, while treatment with andrographolide led to an upregulation of Nrf2 mRNA, the effect was not evident at $8 \mathrm{~h}$ and was only significant by $24 \mathrm{~h}$ (Fig. 1d). In contrast, Nrf2 protein levels increased significantly by $30 \mathrm{~min}$ and the increase was sustained throughout the study timecourse (Fig. 1e), suggesting that andrographolide-mediated increases in Nrf2 protein levels at the acute stage $(<8 \mathrm{~h})$ did not occur via the upregulation of gene expression and de novo protein synthesis but rather may be facilitated by changes in protein level regulation, e.g., activation or turnover. Furthermore, subcellular fractionation revealed that andrographolide promoted Nrf2 accumulation in both nuclear and cytoplasmic compartments (Fig. 1f, g). Significant increases of Nrf2 in nuclear fractions were observed by 30 min which coincided with early upregulation of HO-1 mRNA level (see Fig. 3a). Taken together, these data indicate that andrographolide could induce $\mathrm{HO}-1$ expression acutely by promoting the accumulation of Nrf2.

\section{Andrographolide has no effect on Nrf2 phosphorylation or Keap1}

One of the key regulators of Nrf2 is Keap1, which binds to Nrf2 and promotes its ubiquitination and subsequent degradation by $26 \mathrm{~S}$ proteasome [33]. Phosphorylation of Nrf2 at Ser40 by protein kinase C is known to disrupt Nrf2 binding to Keap1, leading to nuclear accumulation of Nrf2 $[34,35]$. However, while no significant change in pSer40 
Nrf2 immunoreactivity was found, the total Nrf2 increased with andrographolide treatment, thus resulting in a significantly decreased ratio of pSer40 Nrf2 to total Nrf2 (Fig. 2a). The possibility of andrographolide promoting Nrf2 protein accumulation by altering Keap1 levels was also considered; however, Fig. 2b showed no change in Keap1 immunoreactivity with andrographolide treatment. Taken together, the data suggest that andrographolide did not affect Nrf2 accumulation by either Ser 40 phosphorylation-mediated escape from Keap1 or changes to Keap1 itself.

\section{Andrographolide reduced Nrf2 turnover and ubiquitination}

We next studied effects of andrographolide on Nrf2 turnover rate. Treatment of astrocytes with cycloheximide
(CHX), which inhibits de novo protein synthesis, showed that Nrf2 normally had a high turnover rate (half-life of around $10 \mathrm{~min}$, Fig. 2c), in agreement with previous observations [36]. With andrographolide co-treatment, however, the turnover of Nrf2 was significantly decreased, with half-life increased to around $40 \mathrm{~min}$ (Fig. 2c). Since ubiquitination signals for protein degradation, the effect of andrographolide on levels of ubiquitinated Nrf2 was assessed. Interestingly, ubiquitin immunoprecipitation showed higher total Nrf2 (input protein) immunoreactivity without proportional increases in ubiquitinated Nrf2 (Fig. 2d), thus suggesting that altered Nrf2 ubiquitin efficiency may be one possible mechanism by which andrographolide increased Nrf2 stability and subsequent upregulation of effector genes, including HO-1.

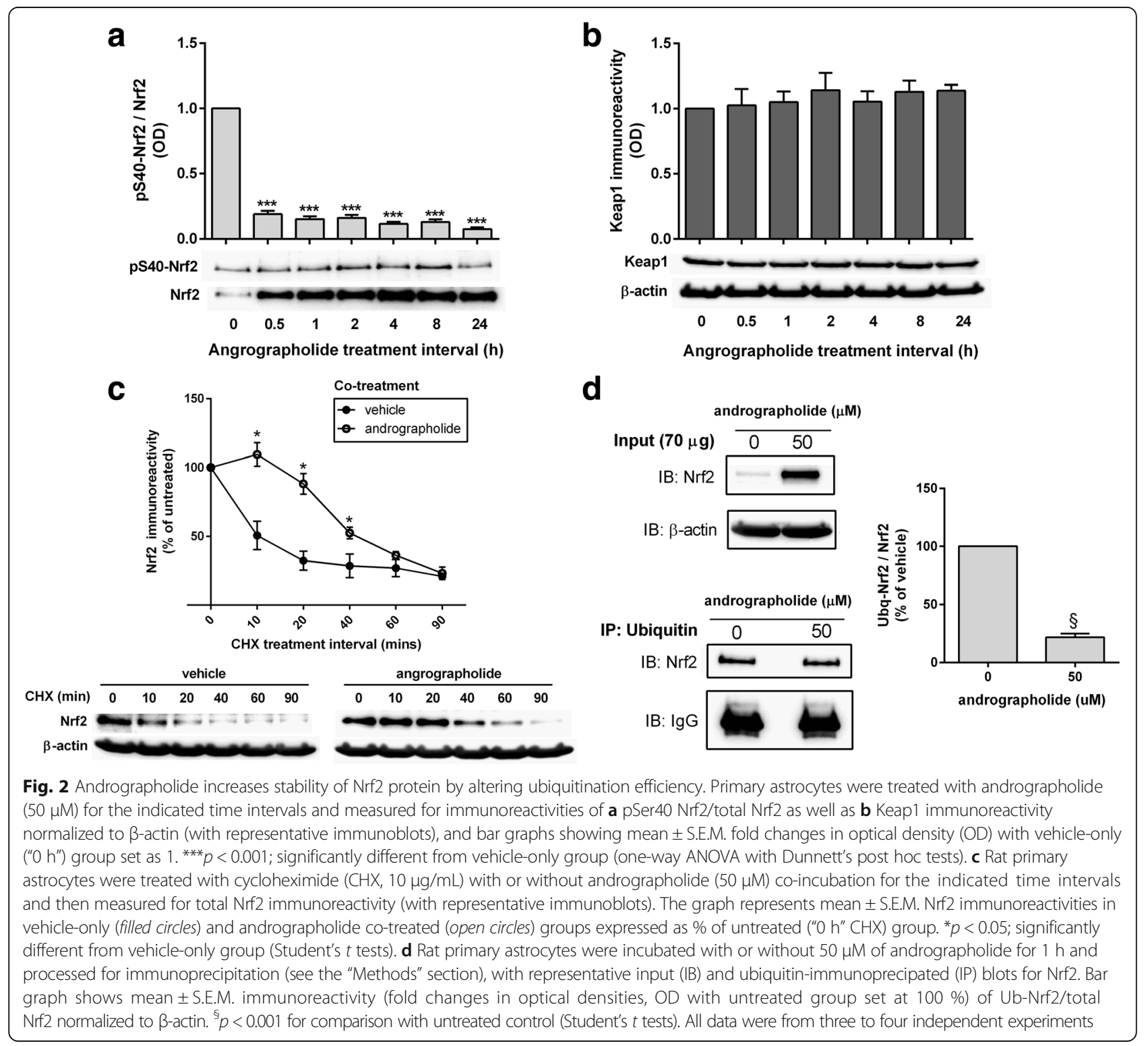


Andrographolide positively regulated HO-1 in astrocytes As Nrf2 levels increased within $1 \mathrm{~h}$ of andrographolide treatment, and therefore likely to regulate $\mathrm{HO}-1$ acutely, we performed RT-PCR and immunoblot time-course experiments and showed that andrographolide $(50 \mu \mathrm{M})$ elevated HO-1 mRNA and protein in a time-dependent manner, with mRNA increase reaching statistical significance from $2 \mathrm{~h}$ while increase in protein was statistically significant from $4 \mathrm{~h}$ (Fig. 3a, b). These data were corroborated qualitatively by immunofluorescence staining which showed increased HO-1 immunoreactivity from around $4 \mathrm{~h}$ after andrographolide treatment (Fig. 3c). Therefore, andrographolide's effects on HO-1 expression may be mediated via a relatively rapid $(<1 \mathrm{~h})$ upregulation of Nrf2. As andrographolide's effects on antioxidative pathways are unlikely to be restricted to $\mathrm{HO}-1$ given Nrf's regulation of multiple gene targets [29], we examined another detoxification and antioxidant molecule, $\mathrm{NAD}(\mathrm{P}) \mathrm{H}$ quinone oxoreductase (Nqo1) [11] and found, indeed, that andrographolide treatment also increased its expression (Additional File 2: Figure S2). This suggests that andrographolide's antioxidant effect is unlikely to be mediated by the upregulation of HO-1 only, and other antioxidant molecules such as Nqo1 are also involved.

\section{Upregulation of Nrf2 and HO-1 by andrographolide is mediated by p38 MAPK- and ERK-dependent pathways} Mitogen-activated protein kinases (MAPKs) such as p38 and extracellular signal-regulated kinase (ERK) are known to facilitate Nrf2 activation and increased expression of Nrf2 target genes [37-40], which led us to speculate whether andrographolide signals to these MAPKs. Interestingly, andrograpolide dose-dependently increased immunoreactivities of phosphorylated (activated) p38 MAPK (Fig. 4a) and p42 ERK (Fig. 4b). Furthermore, pretreatment with inhibitors of p38 MAPK (SB202190) or ERK (PD98059) partially attenuated the upregulation of HO-1 mRNA by andrographolide (Fig. 4c). Similarly, andrographolide-induced Nrf2 accumulation in both cytoplasmic and nuclear fractions was partially attenuated by pretreatment with SB202190 and PD98059 (Figs. 4d, e). These results support the involvement of p38 MAPK and ERK signaling in regulating andrographolide's effects on Nrf2 and HO-1.

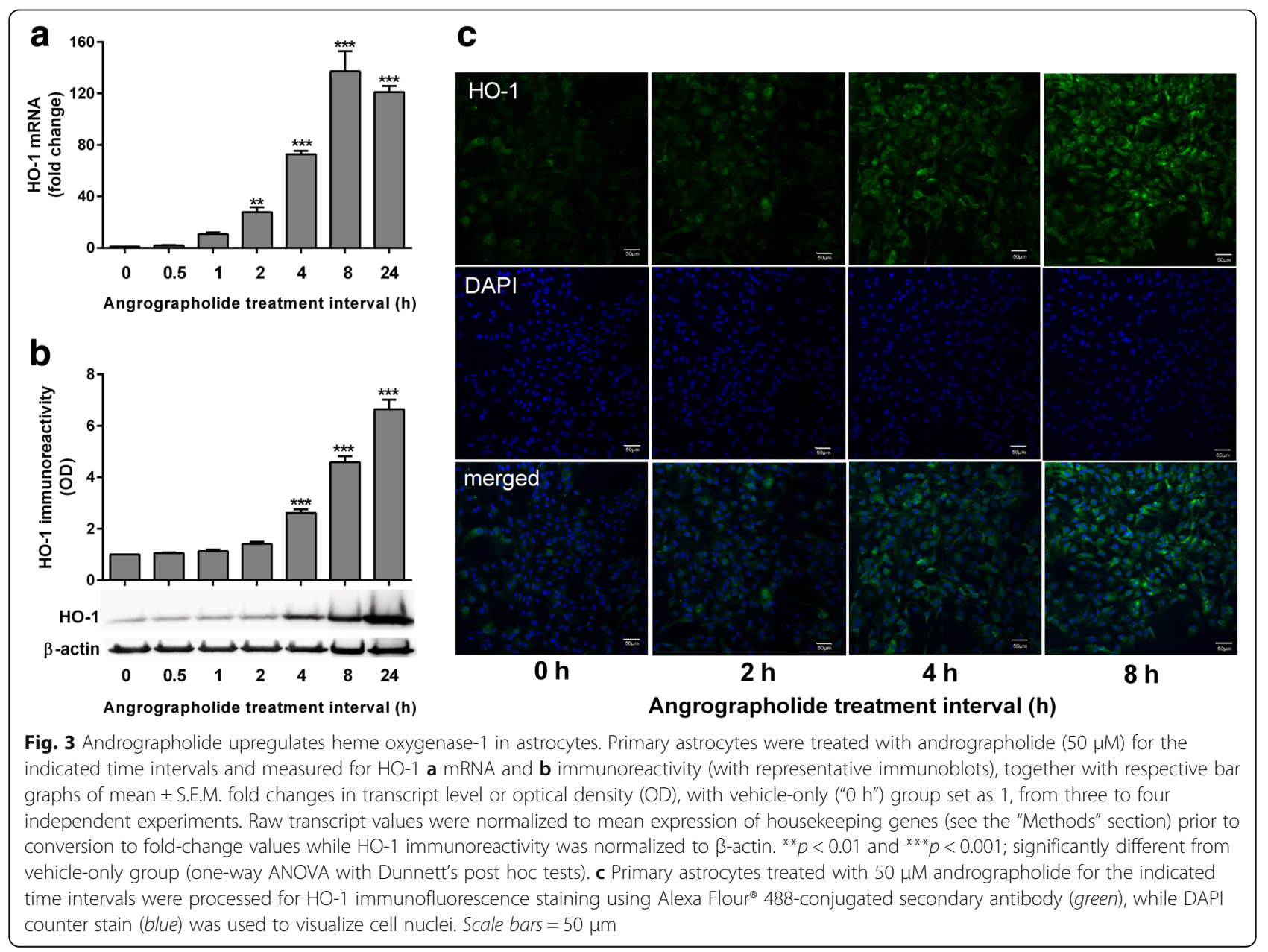




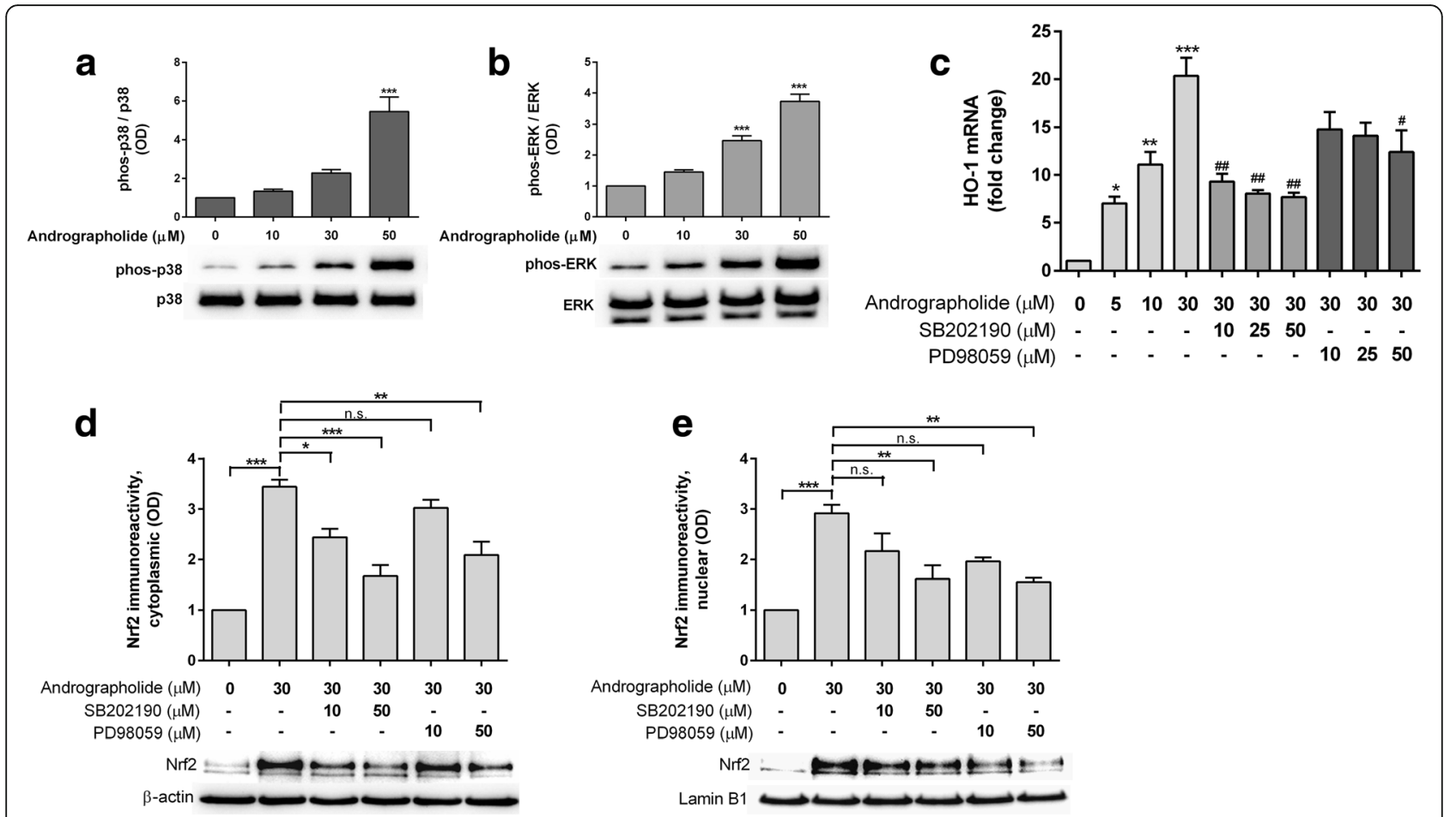

Fig. 4 Andrographolide induces HO-1 expression through p38 MAPK- and ERK-dependent regulation of Nrf2. Effects of andrographolide on a p38 MAPK and $\mathbf{b}$ ERK activation. Primary astrocytes were treated with increasing concentrations of andrographolide for $6 \mathrm{~h}$ and processed for immunobloting. Bar graphs showing mean \pm S.E.M. fold changes in optical density (OD, with vehicle-only "O $\mu \mathrm{M}$ " group set as 1) of phospho-protein normalized to total protein, ${ }^{* * *} p<0.001$; significantly different from vehicle-only group (one-way ANOVA with Dunnett's post hoc tests). c Effects of p38 MAPK and ERK inhibition on HO-1 mRNA expression. Primary astrocytes were pretreated with or without SB202190 (p38 MAPK inhibitor) or PD98059 (MEK/ERK inhibitor) for an hour followed by $4 \mathrm{~h}$ of incubation with andrographolide (with presence of inhibitors) before processing for RT-PCR. Bar graphs are mean \pm S.E.M. fold change in transcript level, with untreated group set as 1. Raw transcript values were normalized to mean expression of housekeeping genes (see the "Methods" section) prior to conversion to fold-change values. ${ }^{*} p<0.05,{ }^{* *} p<0.01$, and ${ }^{* * *} p<0.001$; significantly difference from untreated group (one-way ANOVA followed by Dunnett's post hoc tests.). ${ }^{\prime} p<0.05,{ }^{\# \#} p<0.01$; significantly different from $30 \mu \mathrm{M}$ andrographolide (one- way ANOVA followed by Bonferroni's post hoc tests). $\mathbf{d}$, e Effects of p38 MAPK and ERK inhibition on Nrf2 in the cytosolic and nuclear compartments, respectively. Primary astrocytes were pretreated with PD98059 or SB202190 for $1 \mathrm{~h}$ followed by another $1 \mathrm{~h}$ of incubation with $30 \mu \mathrm{M}$ andrographolide (in the presence of inhibitors). Cytoplasmic and nuclear fractions were separated and processed for immunoblotting. Bar graphs show immunoreactivities (mean \pm S.E.M. fold changes in optical densities, OD, with untreated group set at 1) of Nrf2 normalized to $\beta$-actin (cytoplasmic) or to lamin B1 (nuclear). ${ }^{*} p<0.05,{ }^{* *} p<0.01,{ }^{* * *} p<0.001$; significant pairwise difference and n.s. $=$ not significant $(p>0.05)$ using one-way ANOVA with Bonferroni's post hoc tests. All data were from three to four independent experiments

\section{Discussion}

Neuroinflammation and oxidative stress are highly interconnected processes, and dysregulation of these processes may be pathogenic in various neuroinflammatory and neurodegenerative conditions, leading to recent research efforts to uncover and assess the therapeutic potential of anti-neuroinflammatory and antioxidative compounds $[1-5,11,41]$. To this end, we have previously shown that the anti-neuroinflammatory effects of andrographolide on astrocytes are mediated via the inhibition of NF-kB and c-Jun N-terminal kinase [20, 21]. In this study, our data suggest that andrographolide may also have antioxidative effects in primary astrocytes via the upregulation of $\mathrm{Nrf} 2$, a master regulator of antioxidant responses [29]. In support of this postulate, a previous screening of 54 bioactive compounds identified andrographolide as a more potent Nrf2 activator than tert- butylhydroquinone (tBHQ), an antioxidant frequently used to study Nrf2/ARE activation [42]. Furthermore, we found that HO-1, a known target of Nrf2-regulated transcription and an antioxidative, cytoprotective protein in both CNS and non-CNS cells [8-10], was also upregulated in andrographolide-treated astrocytes. Here, we showed, remarkably, that increases of HO-1 mRNA were observable within $1 \mathrm{~h}$ of andrographolide incubation, closely following the rapid accumulation (within $30 \mathrm{~min}$ ) of Nrf2 (Figs. 1 and 3). We subsequently found that Nrf2 increases were due to reduced Nrf2 ubiquitination efficiency and turnover (Fig. 2). Taken together with observations of increased Nrf2 mRNA from between 8 to $24 \mathrm{~h}$ (Fig. 1a), our data suggest that andrographolide's effect on Nrf2 is biphasic, with an acute effect on protein turnover and a more intermediate effect at the transcript level. Furthermore, while we focused on HO-1 in this study due to 
its established anti-neuroinflammatory and neuroprotective properties in the CNS $[8,10]$, it is very likely that andrographolide will upregulate other antioxidant pathways and molecules via Nrf2 [29], such as Nqo1, another molecule important in the detoxification and prevention of reactive oxygen radical formation (Additional File 2: Figure S2).

Given that the high turnover rate of Nrf2 allows it to be maintained at low, basal levels but be rapidly upregulated in response to oxidative insults [33,36,38], we then studied potential mechanisms underlying andrographolide's effect on Nrf2 turnover, one of which is interaction with Keap1. Under basal conditions, Keap1 binds to the Neh2 domain of Nrf2 and sequesters it in the cytoplasm, acting as a substrate adapter for cullin-3 (Cul3) which, together with other proteins, forms an E3 ubiquitin ligase complex to promote Nrf2 ubiquitination and degradation [33]. Therefore, Nrf2 may potentially escape Keap1-mediated degradation either by downregulating Keap1 or by phosphorylation of Nrf2 at Ser40 which disrupts binding to Keap1 [34, 35]. Although our finding of unchanged Keap1 and reduced rather than increased proportion of pSer40 Nrf2 (Fig. 2a, b) suggested a Keap1-independent mechanism, we cannot rule out the possibility that andrographolide may alter Keap1-Nrf2 association by forming adducts with the thiol groups of reactive cysteine residues on
Keap1, similar to that reported for other Nrf2 inducers [43]. Therefore, confirmatory studies of andrographolide's effects on Keap1 are required.

$\mathrm{Nrf} 2$ is an acidic protein with $\sim 16 \%$ of its total amino acids made up of serine, threonine, and tyrosine residues, making it a probable substrate for several signaling kinases [44]. Emerging evidence indicate that Nrf2 phosphorylation positively regulates its stability, as treatment with phosphatase inhibitors led to Nrf2 hyperphosphorylation, accumulation, and activation of ARE-mediated reporter gene [38]. Moreover, tBHQ and other inducers increased Nrf2 stability and transactivation activity through p38 MAPK and ERK [37-39]. Similarly, we found that andrographolide activated p38 MAPK and ERK in a dose-dependent manner (Fig. 4a, b), while inhibition of p38 MAPK and ERK attenuated andrographolidemediated upregulation of $\mathrm{HO}-1$ transcription as well as Nrf2 accumulation in both cytoplasmic and nuclear compartments (Figs. 4c-e). Our data therefore suggest regulatory roles of ERK and p38 MAPK signaling in Nrf2 activation and $\mathrm{HO}-1$ expression in astrocytes. It is worth noting, however, that Nrf2 and HO-1 induction were only partially attenuated by ERK and p38 MAPK inhibition (Figs. 4c-e), suggesting the involvement of other signaling molecules and pathways. Interestingly, while the two main members of ERK, p42 ERK2, and p44 ERK1 are usually

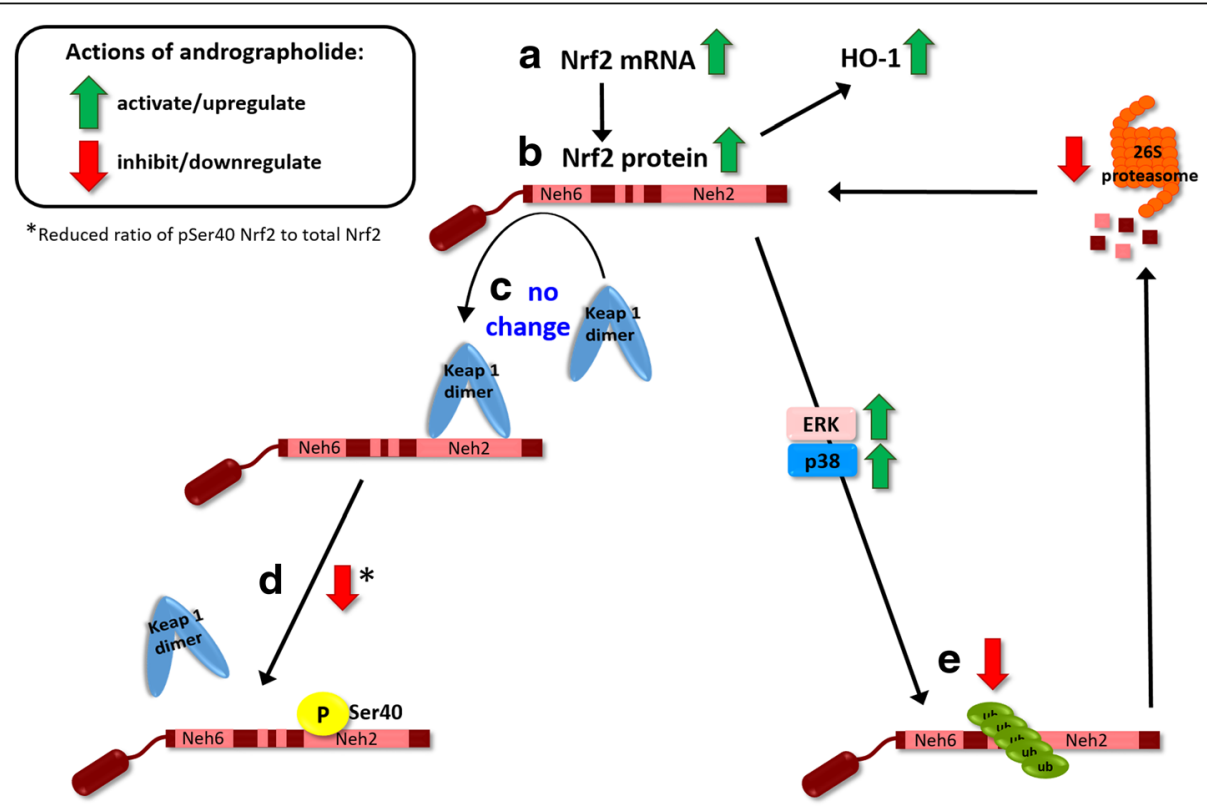

Fig. 5 Andrographolide's effects on Nrf2 and HO-1 in astrocytes. Summary schematic of findings of the current study. Black solid arrows indicate positive regulation, while activation effects of andrographolide are denoted by green arrows, and inhibitory effects are denoted by red arrows. a Andrographolide activates Nrf2 transcription between 8 and 24 h, leading to $b$ increased Nrf2 proteins, which may also accumulate rapidly (within $1 \mathrm{~h}$ ) through a process of altered ubiquitination which $\mathrm{c}$ does not alter Keap1 levels and $d$ does not disrupt Nrf2-Keap1 binding through Ser40 phosphorylation within the Nrf2 Neh2 domain. Instead, andrographolide treatment leads to e reduced Nrf2 ubiquitination efficiency and subsequent 265 proteasomal turnover via p38 MAPK- and ERK-dependent pathways, although it is unclear whether the kinases directly act on Nrf2, or through regulation of other signaling molecules, for, e.g., GSK-3ß. Further studies are required 
co-regulated [45], andrographolide seemed to only activate p42 ERK2 (Fig. 4b), and the mechanism underlying this specificity is unclear at present. Furthermore, there are conflicting reports of andrographolide's effects on signaling kinases, which seemed to depend at least in part on the cell types and time-course studied. For example, we have found that andrographolide inhibits JNK but activates ERK and p38 MAPK in primary astrocytes (see [21] and this study). In contrast, Lee et al. [26] showed in human hepatoma cells that p38, but not ERK, mediated the upregulation of Nrf2 and HO-1 by andrographolide, while in endothelial cells, although andrographolide activated ERK, the induction of $\mathrm{HO}-1$ was dependent on phosphatidylinositol-3 kinase/protein kinase B, rather than on ERK [27]. In the case of the hepatoma cell study [26], the absence of ERK activation may be explained by a relatively short time-course $(2 \mathrm{~h})$, while our time-course experiments found that ERK phosphorylation was only evident after $4 \mathrm{~h}$ of incubation (data not shown). These data suggest that the molecular mechanisms underlying andrographolide's effects are not generalizable across cell types, and studies are needed to characterize different cells/tissues/systems of interest. Lastly, it is at present unclear whether the various signaling kinases activate Nrf2 by direct phosphorylation, or via other signaling molecules or mechanisms. For example, p38 MAPK and ERK can inhibit glycogen synthase kinase-3 $\beta$ (GSK-3 $\beta$ ) phosphorylation of the Neh6 domain of Nrf2, and as a result, attenuate Keap1-independent Nrf2 ubiquitination and degradation via the $\beta$-TrCP/Cul1 E3 ubiquitin ligase complex [44, 46-48]. Further studies are needed to investigate whether this pathway is relevant for astrocytes and other CNS cell types, including microglia and neurons treated with andrographolide. These follow-up studies are especially relevant for microglia, whose activation timescale is faster than astrocytes and provide the initial neuroinflammatory signals which subsequently activate astrocytes [49], and therefore also represent an essential target in anti-neuroinflammatory therapeutics.

\section{Conclusions}

We showed in this study that andrographolide induces the master antioxidant regulator, Nrf2, as well as one of its target genes, HO-1, in primary astrocytes. The induction of Nrf2 seems to be biphasic, with an acute effect (within $1 \mathrm{~h}$ ) of reducing Nrf2 ubiquitination efficiency and subsequent turnover and a longer term effect (between 8 and $24 \mathrm{~h}$ ) of upregulating Nrf2 mRNA, thus enabling the rapid and sustained induction of HO-1. Furthermore, the acute effects did not seem to affect Keap1 levels but may rather be partly dependent on p38 MAPK- and ERK-mediated signaling (Fig. 5). Given the important function of astrocytes in mediating neuroinflammatory responses as well as maintaining redox homeostasis in the neuronal environment, and taking into consideration our previous work on the anti-neuroinflammatory effects of andrgrapholide, the current data provide further insights into the mechanisms underlying the pleiotropic effects of andrographolide on astrocyte-mediated antioxidant and anti-inflammatory responses, and further support the potential therapeutic utility of andrographolide for neurological conditions characterized by inflammation and oxidative stress. However, follow-up studies are needed to (i) further characterize the molecular mechanisms and signaling pathways underlying andrographolide's induction of Nrf2, both acutely and in the longer term; (ii) uncover other antioxidant pathways and molecules which may be affected by andrograpolide; and (iii) study the effects of andrographolide in different CNS cell types, including microglia and neurons, as well as in animal models of diseases.

\section{Additional files}

\begin{abstract}
Additional file 1: Figure S1. Representative immunoblots of Nrf2 in primary astrocytes. a An example of time-point experiment after andrographolide treatment, nuclei fraction (Fig. 1g) and $\mathbf{b}$ an example of ubiquitin immunoprecipitation (IP) after andrographolide treatment (Fig. 2d) with input lysate on the right and IP blot on the left, with indicated molecular weight marker positions. In most cases, two prominent bands above 50 and $100 \mathrm{kDa}$ were visible, and blue arrows indicate the bands selected for analyses (around $110 \mathrm{kDa}$ ) in accordance with Lau et al. [30]. (DOCX $431 \mathrm{~kb}$ )
\end{abstract}

Additional file 2: Figure S2. Andrographolide upregulates $N A D(P) H$ quinone oxoreductase 1 (Nqo1) in astrocytes. Primary astrocytes were treated with andrographolide $(50 \mu \mathrm{M})$ for the indicated time intervals and measured for Nqo1 a mRNA and $\mathbf{b}$ immunoreactivity (with representative immunoblots), together with respective bar graphs of mean \pm S.E.M. fold changes in transcript level or optical density (OD), with vehicle-only ("O h") group set as 1, from 4 independent experiments. Raw transcript values were normalized to mean expression of housekeeping genes (see the "Methods" section) prior to conversion to fold-change values while Nqo1 immunoreactivity was normalized to $\beta$-actin. ${ }^{* *} p<0.001$; significantly different from vehicle-only group (one-way ANOVA with Dunnett's post hoc tests). (DOCX $176 \mathrm{~kb}$ )

\section{Abbreviations}

ANOVA: Analysis of variance; ARE: Antioxidant response element; CHX: Cycloheximide; DAPI: 4',6-Diamidino-2-phenylindole; DMEM: Dulbecco's modified Eagle medium; DMSO: Dimethyl sulfoxide; EDTA: Ethylenediaminetetraacetic acid; ERK: Extracellular signal-regulated kinase; FBS: Fetal bovine serum; GAPDH: Glyceraldehyde-3-phosphate dehydrogenase; HO-1: Heme oxygenase-1; HRP: Horse radish peroxidase; IB: Immunoblot; IP: Immunoprecipitation; Keap1: Kelch-like ECH-associated protein 1; NF-kB: Nuclear factor-kB; Nqo 1: NAD(P)H quinone oxoreductase 1; Nrf2: Nuclear factor erythroid 2-related factor 2; OD: Optical density; PBS: Phosphate-buffered saline; RT-PCR: Reverse transcription polymerase chain reaction; TBP: TATA-binding protein

\section{Acknowledgements}

The authors would like to acknowledge the technical and administrative assistance provided by Xiaoguang Xu and Wee Lee Ting.

\section{Funding}

This study was supported by a grant from the Yong Loo Lin School of Medicine, National University of Singapore (R-184-000-223-133).

Availability of data and materials

Dataset and materials available upon request. 


\section{Authors' contributions}

SYW designed and performed the experiments, analyzed data, and wrote the manuscript. MGKT, PTHW, and DRH designed the experiments and analyzed the data. MKPL initiated the study, designed the experiments, analyzed the data, and wrote the manuscript. All authors have read, revised, and approved the current version of the manuscript.

\section{Competing interests}

All authors declare that they have no competing interests.

\section{Consent for publication}

Not applicable.

\section{Ethics approval and consent to participate}

This study was carried out according to the ARRIVE guidelines of the National Centre for the Replacement Refinement and Reduction of Animals in Research, and approval from the National University of Singapore's Institutional Animal Care and Use Committee (S13-6210) had been obtained prior to study commencement.

\section{Author details}

'Department of Pharmacology, Yong Loo Lin School of Medicine, National University of Singapore, Unit 09-01, Centre for Translational Medicine (MD6), 14 Medical Drive, Kent Ridge 117599, Singapore. ${ }^{2}$ Department of Clinical Research, Singapore General Hospital, Outram, Singapore.

Received: 16 June 2016 Accepted: 15 September 2016

Published online: 23 September 2016

\section{References}

1. Dandekar A, Mendez R, Zhang K. Cross talk between ER stress, oxidative stress, and inflammation in health and disease. Methods Mol Biol. 2015;1292:205-14.

2. Freeman LC, Ting JP. The pathogenic role of the inflammasome in neurodegenerative diseases. J Neurochem. 2016;136 Suppl 1:29-38.

3. Dias $\mathrm{V}$, Junn $\mathrm{E}$, Mouradian MM. The role of oxidative stress in Parkinson's disease. J Parkinsons Dis. 2013;3:461-91.

4. Lozano D, Gonzales-Portillo GS, Acosta S, de la Pena I, Tajiri N, Kaneko Y, Borlongan CV. Neuroinflammatory responses to traumatic brain injury: etiology, clinical consequences, and therapeutic opportunities. Neuropsychiatr Dis Treat. 2015;11:97-106.

5. Taylor JM, Main BS, Crack PJ. Neuroinflammation and oxidative stress: co-conspirators in the pathology of Parkinson's disease. Neurochem Int 2013;62:803-19

6. Belaidi AA, Bush Al. Iron neurochemistry in Alzheimer's disease and Parkinson's disease: targets for therapeutics. J Neurochem. 2016 In Press. doi: 10.1111/jnc.13425.

7. Gandhi S, Abramov AY. Mechanism of oxidative stress in neurodegeneration. Oxid Med Cell Longev. 2012;2012:428010.

8. Jazwa A, Cuadrado A. Targeting heme oxygenase-1 for neuroprotection and neuroinflammation in neurodegenerative diseases. Curr Drug Targets. 2010:11:1517-31.

9. Ryter SW, Alam J, Choi AM. Heme oxygenase-1/carbon monoxide: from basic science to therapeutic applications. Physiol Rev. 2006;86:583-650.

10. Syapin PJ. Regulation of haeme oxygenase-1 for treatment of neuroinflammation and brain disorders. Br J Pharmacol. 2008:155:623-40.

11. Park JS, Jung JS, Jeong YH, Hyun JW, Le TK, Kim DH, Choi EC, Kim HS. Antioxidant mechanism of isoflavone metabolites in hydrogen peroxidestimulated rat primary astrocytes: critical role of hemeoxygenase-1 and NQO1 expression. J Neurochem. 2011;119:909-19.

12. Trendelenburg G, Dirnagl U. Neuroprotective role of astrocytes in cerebral ischemia: focus on ischemic preconditioning. Glia. 2005;50:307-20.

13. Schreiner B, Romanelli E, Liberski P, Ingold-Heppner B, Sobottka-Brillout B, Hartwig T, Chandrasekar V, Johannssen H, Zeilhofer HU, Aguzzi A, et al. Astrocyte depletion impairs redox homeostasis and triggers neuronal loss in the adult CNS. Cell Rep. 2015;12:1377-84.

14. Brambilla L, Martorana F, Rossi D. Astrocyte signaling and neurodegeneration: new insights into CNS disorders. Prion. 2013;7:28-36.

15. Pekny M, Pekna M. Astrocyte reactivity and reactive astrogliosis: costs and benefits. Physiol Rev. 2014;94:1077-98.
16. Sofroniew MV, Vinters HV. Astrocytes: biology and pathology. Acta Neuropathol. 2010;119:7-35.

17. Chao WW, Lin BF. Isolation and identification of bioactive compounds in Andrographis paniculata (Chuanxinlian). Chin Med. 2010;5:17.

18. Panossian A, Davtyan T, Gukassyan N, Gukasova G, Mamikonyan G, Gabrielian E, Wikman G. Effect of andrographolide and Kan Jang-fixed combination of extract SHA-10 and extract SHE-3-on proliferation of human lymphocytes, production of cytokines and immune activation markers in the whole blood cells culture. Phytomedicine. 2002:9:598-605.

19. Lim JC, Chan TK, Ng DS, Sagineedu SR, Stanslas J, Wong WS. Andrographolide and its analogues: versatile bioactive molecules for combating inflammation and cancer. Clin Exp Pharmacol Physiol. 2012;39:300-10.

20. Wong SY, Chan SJ, Wong WS, Wong PT, Lai MK. Andrographolide attenuates interleukin-1b-stimulated upregulation of chemokine CCL5 and glial fibrillary acidic protein in astrocytes. Neuroreport. 2014;25:881-6.

21. Wong SY, Tan MG, Banks WA, Wong WS, Wong PT, Lai MK. Andrographolide attenuates LPS-stimulated up-regulation of C-C and C-X-C motif chemokines in rodent cortex and primary astrocytes. J Neuroinflammation. 2016;13:34.

22. Zhang Z, Lai D, Wang L, Yu P, Zhu L, Guo B, Xu L, Zhou L, Sun Y, Lee SM, Wang $Y$. Neuroprotective effects of the andrographolide analogue AL-1 in the MPP(+)/MPTP-induced Parkinson's disease model in vitro and in mice. Pharmacol Biochem Behav. 2014;122:191-202.

23. Chern CM, Liou KT, Wang YH, Liao JF, Yen JC, Shen YC. Andrographolide inhibits PI3K/AKT-dependent NOX2 and iNOS expression protecting mice against hypoxia/ischemia-induced oxidative brain injury. Planta Med. 2011;77:1669-79.

24. Das S, Gautam N, Dey SK, Maiti T, Roy S. Oxidative stress in the brain of nicotine-induced toxicity: protective role of Andrographis paniculata Nees and vitamin E. Appl Physiol Nutr Metab. 2009:34:124-35.

25. Hsieh CY, Hsu MJ, Hsiao G, Wang YH, Huang CW, Chen SW, Jayakumar T, Chiu PT, Chiu YH, Sheu JR. Andrographolide enhances nuclear factor-kB subunit p65 Ser536 dephosphorylation through activation of protein phosphatase 2A in vascular smooth muscle cells. J Biol Chem. 2011:286:5942-55.

26. Lee JC, Tseng CK, Young KC, Sun HY, Wang SW, Chen WC, Lin CK, Wu YH. Andrographolide exerts anti-hepatitis $C$ virus activity by up-regulating haeme oxygenase-1 via the p38 MAPK/Nrf2 pathway in human hepatoma cells. Br J Pharmacol. 2014;171:237-52.

27. Lu WJ, Lee JJ, Chou DS, Jayakumar T, Fong TH, Hsiao G, Sheu JR. A novel role of andrographolide, an NF-kB inhibitor, on inhibition of platelet activation: the pivotal mechanisms of endothelial nitric oxide synthase/ cyclic GMP. J Mol Med (Berl). 2011;89:1261-73.

28. Bryan HK, Olayanju A, Goldring CE, Park BK. The Nrf2 cell defence pathway: Keap1-dependent and -independent mechanisms of regulation. Biochem Pharmacol. 2013:85:705-17.

29. Ma Q. Role of nrf2 in oxidative stress and toxicity. Annu Rev Pharmacol Toxicol. 2013:53:401-26.

30. Lau A, Tian W, Whitman SA, Zhang DD. The predicted molecular weight of Nrf2: it is what it is not. Antioxid Redox Signal. 2013;18:91-3.

31. Moi P, Chan K, Asunis I, Cao A, Kan YW. Isolation of NF-E2-related factor 2 (Nrf2), a NF-E2-like basic leucine zipper transcriptional activator that binds to the tandem NF-E2/AP1 repeat of the b-globin locus control region. Proc Natl Acad Sci U S A. 1994:91:9926-30.

32. Kang KW, Lee SJ, Park JW, Kim SG. Phosphatidylinositol 3-kinase regulates nuclear translocation of NF-E2-related factor 2 through actin rearrangement in response to oxidative stress. Mol Pharmacol. 2002;62:1001-10.

33. Furukawa M, Xiong Y. BTB protein Keap1 targets antioxidant transcription factor Nrf2 for ubiquitination by the Cullin 3-Roc1 ligase. Mol Cell Biol. 2005;25:162-71.

34. Huang HC, Nguyen T, Pickett CB. Phosphorylation of Nrf2 at Ser-40 by protein kinase $\mathrm{C}$ regulates antioxidant response element-mediated transcription. J Biol Chem. 2002;277:42769-74.

35. Niture SK, Jain AK, Jaiswal AK. Antioxidant-induced modification of INrf2 cysteine 151 and PKC-delta-mediated phosphorylation of Nrf2 serine 40 are both required for stabilization and nuclear translocation of $\mathrm{Nrf} 2$ and increased drug resistance. J Cell Sci. 2009;122:4452-64.

36. Stewart D, Killeen E, Naquin R, Alam S, Alam J. Degradation of transcription factor Nrf2 via the ubiquitin-proteasome pathway and stabilization by cadmium. J Biol Chem. 2003;278:2396-402.

37. Keum YS, Yu S, Chang PP, Yuan X, Kim JH, Xu C, Han J, Agarwal A, Kong AN Mechanism of action of sulforaphane: inhibition of p38 mitogen-activated 
protein kinase isoforms contributing to the induction of antioxidant response element-mediated heme oxygenase-1 in human hepatoma HepG2 cells. Cancer Res. 2006;66:8804-13.

38. Nguyen T, Sherratt PJ, Huang HC, Yang CS, Pickett CB. Increased protein stability as a mechanism that enhances Nrf2-mediated transcriptional activation of the antioxidant response element. Degradation of Nrf2 by the 26 S proteasome. J Biol Chem. 2003;278:4536-41.

39. Zipper LM, Mulcahy RT. Inhibition of ERK and p38 MAP kinases inhibits binding of Nrf2 and induction of GCS genes. Biochem Biophys Res Commun. 2000;278:484-92.

40. Eom HJ, Choi J. Oxidative stress of $\mathrm{CeO} 2$ nanoparticles via p38-Nrf-2 signaling pathway in human bronchial epithelial cell, Beas-2B. Toxicol Lett. 2009;187:77-83.

41. Han Z, Li L, Wang L, Degos V, Maze M, Su H. a7 nicotinic acetylcholine receptor agonist treatment reduces neuroinflammation, oxidative stress, and brain injury in mice with ischemic stroke and bone fracture. J Neurochem. 2014:131:498-508.

42. Wu KC, McDonald PR, Liu J, Klaassen CD. Screening of natural compounds as activators of the keap1-nrf2 pathway. Planta Med. 2014;80:97-104.

43. Dinkova-Kostova AT, Holtzclaw WD, Cole RN, Itoh K, Wakabayashi N, Katoh Y, Yamamoto M, Talalay P. Direct evidence that sulfhydryl groups of Keap1 are the sensors regulating induction of phase 2 enzymes that protect against carcinogens and oxidants. Proc Natl Acad Sci U S A. 2002;99:11908-13.

44. Rojo Al, Medina-Campos ON, Rada P, Zuniga-Toala A, Lopez-Gazcon A Espada S, Pedraza-Chaverri J, Cuadrado A. Signaling pathways activated by the phytochemical nordihydroguaiaretic acid contribute to a Keap1independent regulation of Nrf2 stability: role of glycogen synthase kinase-3. Free Radic Biol Med. 2012;52:473-87.

45. Houslay MD, Kolch W. Cell-type specific integration of cross-talk between extracellular signal-regulated kinase and CAMP signaling. Mol Pharmacol. 2000;58:659-68

46. Thornton TM, Pedraza-Alva G, Deng B, Wood CD, Aronshtam A, Clements JL, Sabio G, Davis RJ, Matthews DE, Doble B, Rincon M. Phosphorylation by p38 MAPK as an alternative pathway for GSK3b inactivation. Science. 2008;320:667-70

47. Ding Q, Xia W, Liu JC, Yang JY, Lee DF, Xia J, Bartholomeusz G, Li Y, Pan Y, Li Z, et al. Erk associates with and primes GSK-3ß for its inactivation resulting in upregulation of beta-catenin. Mol Cell. 2005;19:159-70.

48. Rada P, Rojo Al, Chowdhry S, McMahon M, Hayes JD, Cuadrado A. SCF/B$\operatorname{TrCP}$ promotes glycogen synthase kinase 3-dependent degradation of the Nrf2 transcription factor in a Keap1-independent manner. Mol Cell Biol. 2011:31:1121-33.

49. Cherry JD, Olschowka JA, O'Banion MK. Neuroinflammation and M2 microglia: the good, the bad, and the inflamed. J Neuroinflammation. 2014;11:98.

\section{Submit your next manuscript to BioMed Central and we will help you at every step:}

- We accept pre-submission inquiries

- Our selector tool helps you to find the most relevant journal

- We provide round the clock customer support

- Convenient online submission

- Thorough peer review

- Inclusion in PubMed and all major indexing services

- Maximum visibility for your research

Submit your manuscript at www.biomedcentral.com/submit

) Biomed Central 